\title{
Ecology and Diversity of Diatoms in Kuttanadu Paddy Fields in Relation to Soil Regions, Seasons and Paddy-Growth-Stages
}

\author{
Dhanya Vijayan $^{1} \&$ J. G. Ray ${ }^{2}$ \\ ${ }^{1}$ Research Scholar, Environment Science Research Lab, St. Berchmans College, Changanacherry, Kerala, India \\ ${ }^{2}$ Professor, School of Biosciences, Mahatma Gandhi University, Kottayam, Kerala, India \\ Correspondence: J. G. Ray, School of Biosciences, Mahatma Gandhi University, Kottayam, Kerala, India. \\ E-mail: jgray@mgu.ac.in
}

Received: January 18, 2016 Accepted: March 17, 2016 Online Published: April 21, 2016

doi:10.5539/jps.v5n2p7

URL: http://dx.doi.org/10.5539/jps.v5n2p7

\begin{abstract}
This assessment of ecology and diversity of Diatoms in Kuttanadu, is continuation of the previous publications of Green-algae and Blue-green-algae of the same region; all the three investigations were carried out simultaneously. The unique Kuttanadu wetlands $\left(90^{\circ} 17^{\prime} \mathrm{N}\right.$ to $90^{\circ} 40^{\prime} \mathrm{N} ; 760^{\circ} 19^{\prime} \mathrm{E}$ to $\left.760^{\circ} 33^{\prime} \mathrm{E}\right)$, a well known 'Rice bowl' of Kerala, spread over 53,639 hectares, is located in Alapuzha District of Kerala, India. This wetland was once known for its high biodiversity, but currently severely affected by intensive green-revolution activities of past few decades. Main objective of the present report is to explain the diversity and ecological status of Diatoms in relation to the environment conditions currently existing in the region. Duration of the study was from December 2009 to October 2010. Soil samples of three different soil-types in two different crop-seasons, at two diverse crop-growth-stages are analyzed and compared. Altogether 120 composite soil samples randomly collected from the broad wetlands are analyzed. A rich community of Diatoms, of 40 species is found out. The order Naviculales is observed as the dominant Diatom (40\%) in these paddy wetlands. Ecological parameters of Diatoms were found highest in the Lower Kuttanadu soil region, during Virippu season, at the seedling- stage of the crop, whereas the lowest value for most of the parameters were observed in Kayal soils during Puncha season at the seedling-stage. Apart from the specific soil factors influencing Diatom population characteristics in the region, crop seasons and soil phosphorus are found to have significant influence on Diatoms in this wetland paddy soils.
\end{abstract}

Keywords: diatoms, paddy-wetland, crop-seasons, soil-regions, paddy-growth-stages

\section{Introduction}

This is the third in the series of reports on ecology and diversity of algae of Kuttanadu wetlands, the first two parts, one on green-algae and the other on blue-green-algae are already published (Vijayan \& Ray, 2015a, 2015b).

Diatoms are one of the most diverse groups of algae, estimated to be more than 100,000 species, with cosmopolitan distribution (Demirbas \& Demirbas, 2011). Uniqueness of Diatoms is that they are not just universally abundant group of algae, but are highly ecologically diverse (Jena, Ratha, \& Adhikary, 2006). Moreover, they are one of the most successful groups of photosynthetic eukaryotes (Muruganantham, Gopalakrishnan, Chandrasekharan, \& Jayachandran, 2012). Seasonal change of diatom community in flooded paddy soil environments is a widely discussed topic worldwide.

Another major significance of the study of Diatoms is that they are well known bio-indicators, capable of providing specific information on varieties of specific environment characteristics (Seirieyssol, Chatelard, \& Cubizolle, 2010) of specific habitats. Some of them are highly tolerant to harsh environmental conditions, while some others are highly sensitive to soil conditions such as desiccation, freezing and abrupt heating (Souffrean, 2010). Such characteristics make the group ideal ecological indicators (Mazumder, 2012).

Like all other algae, diatoms also contribute significantly to carbon sequestration; but the role of diatoms in the recycling of silica is highly specific (Kesic, Tuney, Zerben, Guden \& Sukatar, 2013). Diatoms constitute a fundamental link between primary and secondary production in aquatic systems. Therefore, the major ecological importance of diatoms is as a component in the food chains of specific aquatic species. Influence of diatoms on 
the mechanical, hydraulic, and physico-chemical properties of soil is also well known.

Many species of Diatoms are now well known for the bio-oil content (McGinnis, Dempster, \& Sommerfeld, 1997 ) in the cells. Some species of diatoms are already commercially cultivated as oil yielding resources and other agents of bio-production (Wanga et al., 2014; Xue et al., 2015).

Therefore, assessment of the diversity of diatoms of any specific ecosystem, especially the ecological details of them in relation to specific environment conditions, has universal significance. Such investigations may also be highly useful in the identification of economically valuable species of diatoms. Discovery of the ecological details of such valuable diatoms have applications in the commercial cultivation of them as bio-resources.

It is true that Diatom diversity in many parts of India have been well documented by various investigators (Gandhi, 1956; Pandey \& Pandey, 1980; Mohan, 1987; Nautiyal \& Verma, 1997; Venkataraman, 2005; Jena et al., 2006; Seirieyssol et al., 2010; Pareek, Singh, \& Singh, 2011; Muruganantham et al., 2012). However, reports of diatom community in Indian paddy fields are quite rare. This is the first report of diatom community of Kuttanadu wetland paddy fields; the uniqueness of the present report is that it provides detailed ecological description of the wet soil diatom community in relation to three different types of wetland soils, two different crop-seasons and at two different paddy-growth-stages.

\section{Material and Methods}

Study area, sampling sites and methodology of sampling were the same as that of the previous reports on green algae and blue-green algae (Vijayan \& Ray, 2015a, 2015b) as all these three reports are based on the same soil sampling, and the studies were carried out simultaneously.

\subsection{Study Area}

Kuttanadu $\left(9^{0} 17^{\prime}-9^{0} 40^{\prime} \mathrm{N}\right.$ and $\left.76^{0} 19^{\prime}-6^{0} 33^{\prime} \mathrm{E}\right)$ is the delta of five eastward flowing rivers from the Western Ghats to the Arabian Sea. This delta is seen on the south eastern boundary of the Vembanadu Lake, a 'Ramsar site', which opens to the sea at Kochi. It is the well known unique 'Rice bowl' of Kerala spread over 53,639 hectares (Sudhikumar, Mathew, Sunish, \& Sebastian, 2005). The Kuttanadu paddy fields are below sea-level

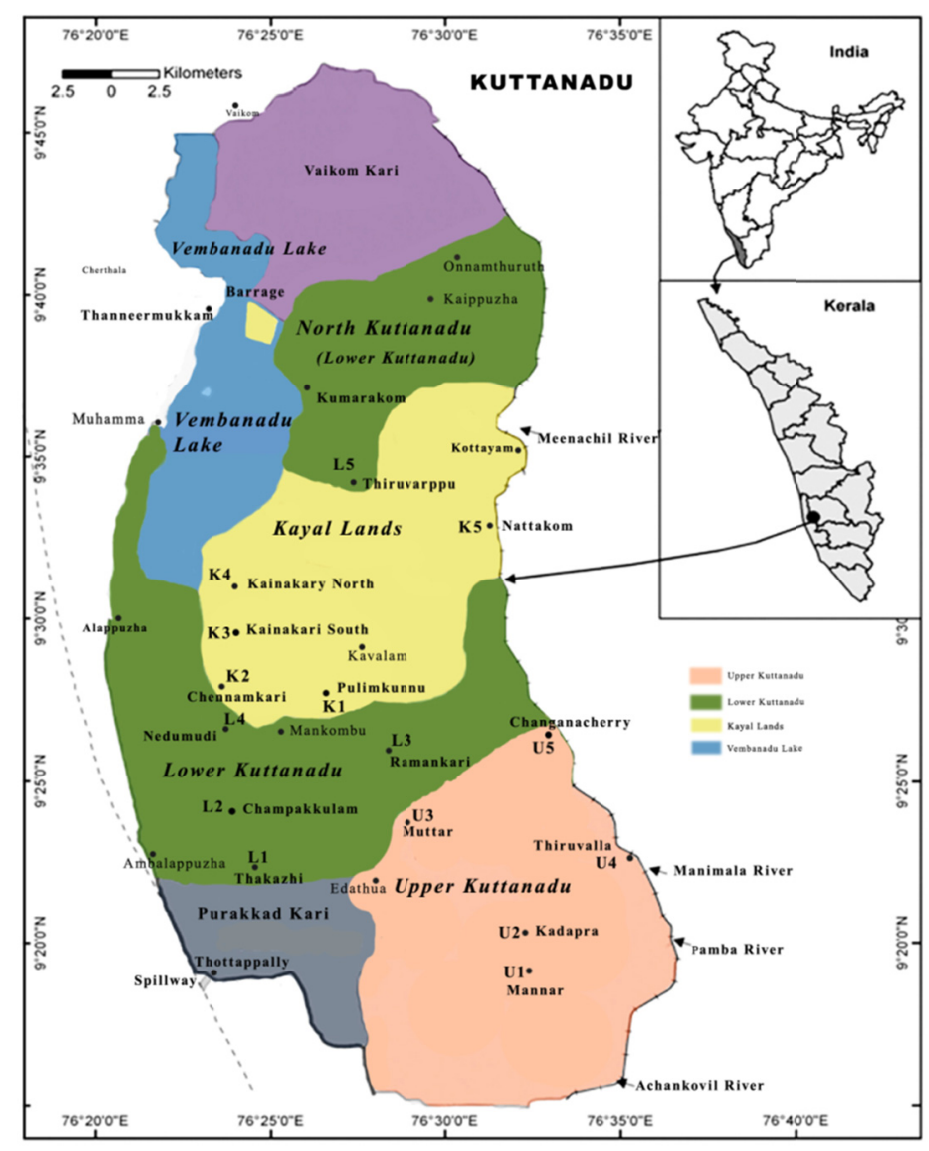

Figure 1. Map of Kuttanadu showing the three different kinds of soils in the broad region and the sampling sites 
wetlands where cultivation is carried out after pumping out the excess water of the broad fields, which during the season of paddy cultivation remain separated from the surrounding backwaters by artificially reclaimed protective 'barrages'. Majority of the fields are cultivated twice a year. The non-monsoon crop, 'Puncha', is the main crop, which is sown in November or December and harvested by the end of March. The additional monsoon crop, 'Virippu' is sown in May to the end of June and harvested in September or October. Upper Kuttanadu, Lower Kuttanadu and Kayal lands are the three soil regions of Kuttanadu. Three kinds of soils reported from the area are 'Karappadom' (river-borne alluvial soils found mostly in the Upper Kuttanadu), 'Kari' (deep black in colour with high proportion of organic matter, characterized by acidity and salinity found mainly in the Lower Kuttanaduand, and rarely in some parts of the upper Kuttanadu zones) and 'Kayal' (the reclaimed shallow beds of Vembanadu Lake, mainly silt-loam to silt-clay-loam) soils (Pillai, Ponniah, Vincent, \& David, 1983). All the geographic and other details of the three soils regions are well described in the previous reports (Ray, Dhanya, \& Binoy, 2014). The Kuttanadu is a unique wetland ecological zone with high biodiversity, especially of indigenous fishes, but quite destroyed by intensive green-revolution efforts of past few decades in the area. It is believed that once up on a time, the brackish water environments of Kuttanadu promoted a rich diversity of mangroves and provided ideal habitats for diverse flora and fauna.

\subsection{Soil Sampling Procedure}

Soil samples were systematically collected from all the three soil regions in two different paddy cultivating seasons of a year; in each season, samples of two different paddy growth stages were collected. At each site two broad specific paddy fields located at 1-2 km intervals were randomly selected for sampling. In each specific field, several plots (5-10) of about $10 \mathrm{~m}^{2}$ areas were randomly identified for sampling. Several soil samples (5-10 from a plot) from the upper $1-5 \mathrm{~cm}$ soil layer from all these plots were collected and put together in a sterile cotton bag using aseptic implement. Repetitive samples put in each cotton bag from different plots of a single paddy field on air drying were mixed thoroughly to a composite sample. There were five sites in a soil region. Since there were five sites, two sampling fields for each site and two crop seasons (the Puncha - summer crop of December 2009 to March 2010; the Virippu - the additional crop of June 2010 to October 2010) to sample and two crop-growth stages (seedling stage and panicle stage) at each season to sample, altogether, 40 composite soil samples $(5 \times 2 \times 2 \times 2)$ were collected from each soil region. Altogether, there were 120 composite soil samples ( 3 x 40) representing the three soil regions of Kuttanad - the 'Upper Kuttanadu' or 'Karappadom', the 'Lower Kuttanadu or 'Kari' ' and the 'Kayal' zones for the soil study and for diatom studies.

\subsection{Physico-chemical Analyses of Soil Samples}

Details of physico-chemical analyses of soils are already reported (Ray, Dhanya, \& Binoy, 2014). Physico chemical analyses of soils were completed within two weeks after the collection. Determination of $\mathrm{pH}$ (1water: 2.5 soil), was done using $\mathrm{pH}$ meter (Systronics 324) organic carbon (Walkley \& Black, 1934) total nitrogen (Microkjeldahl method), and available Phosphorus (Bray \& Kurtz, 1945), Potassium (Flame photometric method), Calcium and Magnesium (Atomic Absorption Spectrometry, Perkin Elmer A Analyst-400) were done by standard analytical procedures (Jackson, 1973).

\subsection{Study of Diatom Flora}

Methods of Van de Vijver and Beyens (1998) and Jena et al. (2006) were used in the microscopic examination of soil samples for diatoms. Microphotograph of each specimen was taken using Olympus digital Camera attached to light microscope. Taxonomical descriptions of Gandhi (1956), Sarode and Kamat (1984), Taylor, Archibald and Harding (2007) were followed in the identification of species.

\subsection{Scanning Electron Microscopic (SEM) Analyses of Diatoms}

Soil samples for diatom analyses were placed in air tight plastic bags to prevent moisture loss from the algae and the samples were repeatedly sprayed with iodine to preserve them. The samples were then sieved through a 75 micron nylon mesh. The SEM studies of cleaned specimens were carried out after ion coating in high vacuum mode Jeol make model- JSM 6390 LA machine. The specimen were fixed to carbon tapes $(1 \mathrm{x} 15 \mathrm{~cm})$ placed on aluminium stubs, coated with gold and underwent magnetron sputtering by auto fine coater - Jeol JFC-1600. The accelerating voltage was $20 \mathrm{KV}$. SEM analyses were carried out in the laboratory of the sophisticated test and Instrumentation Centre (STIC), University of Science and Technology, Cochin.

\subsection{Environment Relationships and Population Dynamics of Diatoms}

Correlation of diatoms to environmental factors such as region, season, and physiochemical soil parameters are described. Method of Dey, Tayung and Bastia (2010) is followed in the study of relative abundance of species in the community. Diversity index (Shannon Wiener Index) and species richness of all species of diatoms in 
different seasons were worked out as per Shannon \& Wiener, (1949). Species richness was calculated following the method of Whittaker, (1977). Classification of taxa was done as per the classification of Guiry \& Guiry, (2012).

\section{Results}

Altogether 40 species of diatoms (Table 1; Figures 2 \& 3) belonging to 19 genera have been recorded from Kuttanadu paddy fields during the entire study period (2009-10). Diatoms collected belonged to nine orders, which are Melosirales (1genus, 2 species), Thalassiorales (1 genus, 1 species), Fragilariales (3genera, 4 species), Thalassiophysales (1 genus, 1species), Naviculaes (8 genera, 16 species), Bacillariales (2 genera, 12 species), Cymbellales (2 genera, 1 species), Eunotiales (1 genera, 1 species) and Achanthales (1 genus, 1 species).

Table 1. Occurrence of diatoms in Kuttanadu paddy fields in different regions, seasons and paddy-growth-stages: (R1-Upper Kuttanadu, R2-Lower Kuttanadu and R3-Kayal Lands; S1-Pre-monsoon (Puncha) crop and S2-Monsoon (Virippu) crop; G1-Seedling-growth-stage and G2-Mature-growth-stage); Symbols (+) presence of the species, $(-)$ absence of the species

\begin{tabular}{|c|c|c|c|c|c|c|c|c|c|c|c|c|c|}
\hline \multirow{3}{*}{ No } & \multirow{3}{*}{ Diatom species } & \multicolumn{4}{|l|}{$\mathrm{R} 1$} & \multicolumn{4}{|l|}{$\mathrm{R} 2$} & \multicolumn{4}{|l|}{ R3 } \\
\hline & & \multicolumn{2}{|l|}{ S1 } & \multicolumn{2}{|l|}{$\mathrm{S} 2$} & \multicolumn{2}{|l|}{$\mathrm{S} 1$} & \multicolumn{2}{|l|}{$\mathrm{S} 2$} & \multicolumn{2}{|l|}{ S1 } & \multicolumn{2}{|l|}{$\mathrm{S} 2$} \\
\hline & & G1 & G2 & G1 & G2 & G1 & G2 & G1 & G2 & G1 & G2 & G1 & G2 \\
\hline & Order: Melosirales & & & & & & & & & & & & \\
\hline 1 & Melosira variansAgardh & + & + & + & + & + & + & + & + & + & + & + & + \\
\hline \multirow[t]{2}{*}{2} & $\begin{array}{l}\text { Melosira italica (Ehrenberg) } \\
\text { Kutzing }\end{array}$ & + & + & + & + & + & + & + & + & + & + & + & + \\
\hline & Order: Thalassiosirales & & & & & & & & & & & & \\
\hline \multirow[t]{2}{*}{3} & $\begin{array}{l}\text { Cyclotella } \\
\text { meneghinianaKutzing }\end{array}$ & + & + & + & + & + & + & + & + & + & + & + & + \\
\hline & Order: Fragilariales & & & & & & & & & & & & \\
\hline 4 & $\begin{array}{l}\text { Fragilaria capucina } \\
\text { Desmazieres }\end{array}$ & + & + & + & + & + & + & + & + & + & + & + & + \\
\hline 5 & $\begin{array}{l}\text { Staurosira elliptica } \\
\text { (Schumann) Williams \& } \\
\text { Round }\end{array}$ & + & + & + & + & + & + & + & + & + & + & + & + \\
\hline 6 & $\begin{array}{l}\text { Synedraulna (Nitzsch) } \\
\text { Ehrenberg }\end{array}$ & + & + & + & + & + & + & + & + & + & + & + & + \\
\hline \multirow[t]{2}{*}{7} & $\begin{array}{l}\text { Synedratabulate (C.Agardh) } \\
\text { Kutzing }\end{array}$ & + & + & + & + & + & + & + & + & + & + & + & + \\
\hline & Order: Thalassiophysales & & & & & & & & & & & & \\
\hline \multirow[t]{2}{*}{8} & $\begin{array}{l}\text { Amphora pediculus(Kutzing) } \\
\text { Grunowex. A. Schmidt }\end{array}$ & + & + & + & + & + & + & + & + & + & + & + & + \\
\hline & Order: Naviculales & & & & & & & & & & & & \\
\hline 9 & $\begin{array}{l}\text { Navicula capitatoradiata } \\
\text { Germain }\end{array}$ & + & + & + & + & + & + & + & + & + & + & + & + \\
\hline 10 & $\begin{array}{l}\text { Navicula cuspidata(Kutzing) } \\
\text { Kutzing }\end{array}$ & + & + & + & + & + & + & + & + & + & + & + & + \\
\hline 11 & $\begin{array}{l}\text { Navicula ranomafenensis } \\
\text { Kutzing }\end{array}$ & + & + & + & + & + & + & + & + & + & + & + & + \\
\hline 12 & $\begin{array}{l}\text { Navicula reinhardtii } \\
\text { (Grnow) Grunow }\end{array}$ & + & + & + & + & + & + & + & + & + & + & + & + \\
\hline 13 & $\begin{array}{l}\text { Navicula salinarum } \\
\text { Grunow }\end{array}$ & + & + & + & + & - & - & + & + & + & + & + & + \\
\hline 14 & Navicula pupula Kutzing & + & + & + & + & - & + & + & + & - & - & + & + \\
\hline 15 & $\begin{array}{l}\text { Navicula viridula(Kutzing) } \\
\text { Ehrenberg }\end{array}$ & + & + & + & + & - & - & + & - & - & - & + & + \\
\hline 16 & $\begin{array}{l}\text { Navicula viridis(Nitzsch) } \\
\text { Kutzing }\end{array}$ & + & + & + & + & - & - & + & - & - & - & + & + \\
\hline 17 & $\begin{array}{l}\text { Navicula } \quad \text { lanceolata } \\
\text { Ehrenberg }\end{array}$ & + & + & + & + & - & - & + & + & - & - & + & + \\
\hline 18 & Capartogramma crucicula & + & - & + & + & + & + & + & + & - & + & + & + \\
\hline
\end{tabular}




\begin{tabular}{|c|c|c|c|c|c|c|c|c|c|c|c|c|c|}
\hline \multirow{3}{*}{ No } & \multirow{3}{*}{ Diatom species } & \multicolumn{4}{|l|}{$\mathrm{R} 1$} & \multicolumn{4}{|l|}{$\mathrm{R} 2$} & \multicolumn{4}{|l|}{ R3 } \\
\hline & & \multicolumn{2}{|l|}{$\mathrm{S} 1$} & \multicolumn{2}{|l|}{$\mathrm{S} 2$} & \multicolumn{2}{|l|}{$\mathrm{S} 1$} & \multicolumn{2}{|l|}{$\mathrm{S} 2$} & \multicolumn{2}{|l|}{$\mathrm{S} 1$} & \multicolumn{2}{|l|}{$\mathrm{S} 2$} \\
\hline & & G1 & $\mathrm{G} 2$ & G1 & $\mathrm{G} 2$ & G1 & G2 & G1 & G2 & G1 & G2 & G1 & $\mathrm{G} 2$ \\
\hline & \multicolumn{13}{|l|}{ Grunow (R.Ross) } \\
\hline 19 & $\begin{array}{l}\text { Craticula cuspidate } \\
\text { (Kutzing) D.G.Mann }\end{array}$ & + & + & + & + & - & - & + & + & - & - & + & + \\
\hline 20 & $\begin{array}{l}\text { Craticula halophila } \\
\text { (Kutzing) D.G.Mann }\end{array}$ & + & + & + & + & + & + & + & + & + & - & - & - \\
\hline 21 & $\begin{array}{l}\text { Frustulia vulgaris } \\
\text { (Thwaites) De Toni }\end{array}$ & + & + & + & + & + & + & + & + & + & + & + & + \\
\hline 22 & Nedium affine (Ehrn) Pfitzer & + & - & + & + & + & + & + & + & + & + & + & + \\
\hline 23 & $\begin{array}{l}\text { Diadesmus confervaceae } \\
\text { (Kutzing) DG Mann }\end{array}$ & + & + & + & + & + & + & + & + & + & + & + & + \\
\hline \multirow[t]{2}{*}{24} & $\begin{array}{l}\text { Sellaphora ulna (Nitzsch) } \\
\text { Ehrenberg }\end{array}$ & + & - & + & + & + & + & + & + & + & + & + & + \\
\hline & Order: Bacillariales & & & & & & & & & & & & \\
\hline 25 & $\begin{array}{l}\text { Pinnularia acrosphaeria W } \\
\text { Smith }\end{array}$ & + & + & + & + & + & + & + & + & + & + & + & + \\
\hline 26 & $\begin{array}{l}\text { Pinnularia biceps } \mathrm{W} . \\
\text { Gregory }\end{array}$ & + & + & + & + & + & + & + & + & - & - & + & + \\
\hline 27 & $\begin{array}{l}\text { Pinnularia brauni (Grunow) } \\
\text { Cleve }\end{array}$ & + & + & + & + & + & + & + & + & - & - & + & + \\
\hline 28 & Pinnularia gibba Ehrenberg & + & + & + & + & + & + & + & + & + & + & + & + \\
\hline 29 & $\begin{array}{l}\text { Pinnularia viridiformis } \\
\text { Krammer }\end{array}$ & + & + & + & + & + & + & + & + & + & + & + & + \\
\hline 30 & Nitzschia reversa W Smith & + & + & + & + & + & + & + & + & + & + & + & + \\
\hline 31 & $\begin{array}{l}\text { Nitzschia sigmoidea } \\
\text { (Nitzsch) W.Smith }\end{array}$ & + & + & + & + & + & + & + & + & + & + & + & + \\
\hline 32 & Nitzschia clausii Hantzsch & + & + & + & + & + & + & + & + & + & + & + & + \\
\hline 33 & $\begin{array}{l}\text { Nitzschia filiformis (W } \\
\text { Smith) Vanurk }\end{array}$ & + & + & + & + & + & + & + & + & + & + & + & + \\
\hline 34 & Nitzschia linearis W Smith & + & + & + & + & + & + & + & + & + & + & + & + \\
\hline 35 & Nitzschia nana Grunow & + & + & + & + & + & + & + & + & + & + & + & + \\
\hline \multirow[t]{2}{*}{36} & $\begin{array}{l}\text { Nitzschia obtusa } \\
\text { Rabenhorst }\end{array}$ & + & + & + & + & + & + & + & + & + & + & + & + \\
\hline & Order: Cymbellales & & & & & & & & & & & & \\
\hline 37 & Cymbella kolbei Hustedt & + & + & + & + & + & + & + & + & + & + & + & + \\
\hline \multirow[t]{2}{*}{38} & $\begin{array}{l}\text { Gomphonema acuminatum } \\
\text { Ehr }\end{array}$ & - & + & + & + & + & + & + & + & - & + & + & + \\
\hline & Order: Eunotiales & & & & & & & & & & & & \\
\hline \multirow[t]{2}{*}{39} & $\begin{array}{l}\text { Eunotia bilunaris } \\
\text { (Ehrenberg) Mills }\end{array}$ & + & + & + & + & + & + & + & + & + & + & + & + \\
\hline & Order:Achnanthales & & & & & & & & & & & & \\
\hline \multirow[t]{2}{*}{40} & $\begin{array}{l}\text { Achnanthidium } \\
\text { minutissimum (Kutzing) } \\
\text { Czarnecki }\end{array}$ & - & - & - & - & + & + & + & + & - & - & - & - \\
\hline & Total species & 35 & 36 & 39 & 39 & 33 & 35 & 40 & 38 & 29 & 30 & 38 & 38 \\
\hline
\end{tabular}



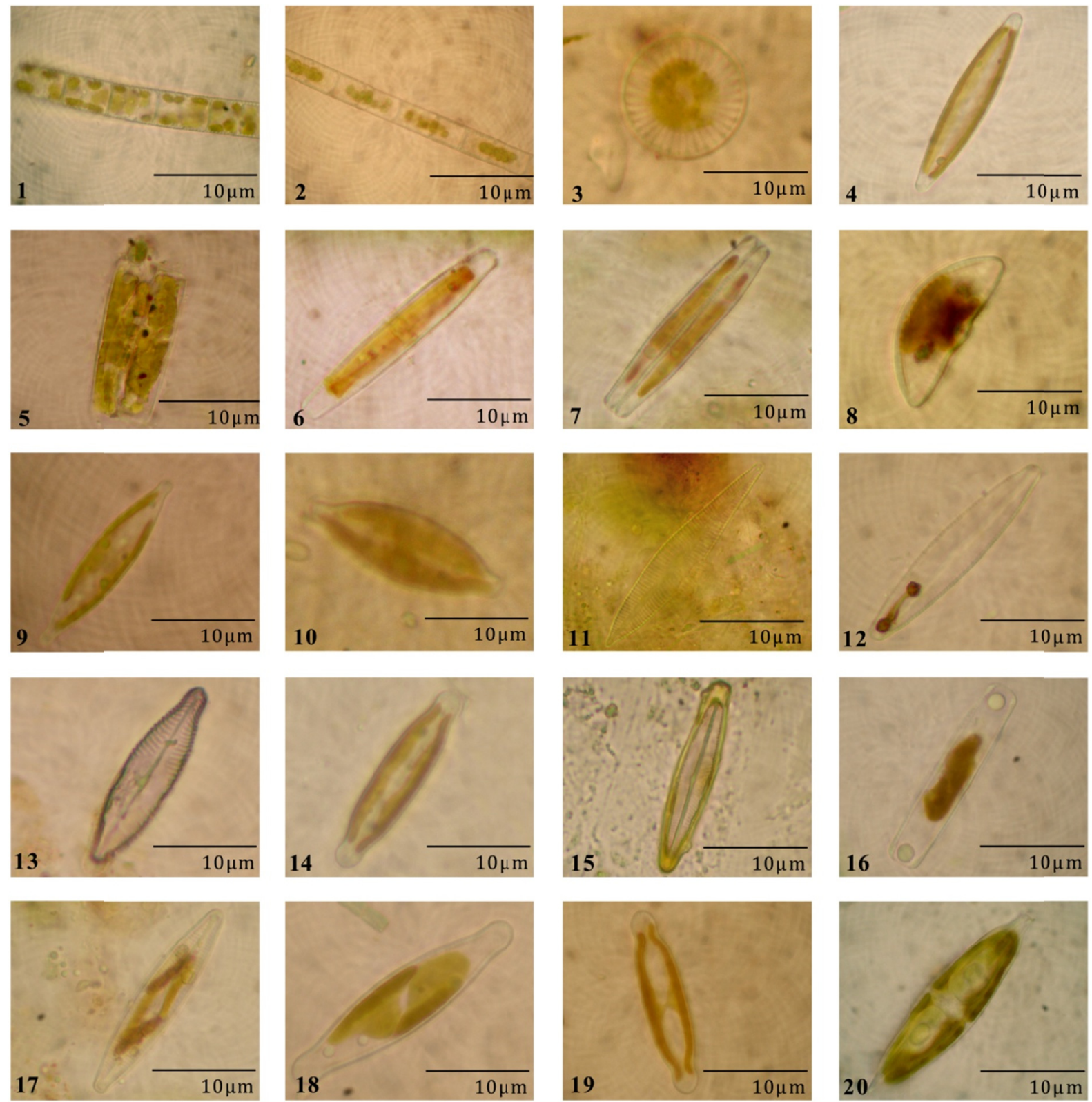

Figure 2. Diatoms $1-20$

1. Melosira varians, 2. Melosira italica, 3. Cyclotella meneghiniana, 4. Fragilaria capucina, 5. Staurosira construens, 6. Synedra ulna, 7. Synedra tabulate, 8. Amphora pediculus, 9. Navicula capitatoradiata, 10. Navicula cuspidate, 11. Navicula ranomafenensis, 12. Navicula reinhardtii, 13. Navicula salinarum, 14. Navicula pupula, 15. Navicula viridula, 16. Navicula viridis, 17. Navicula lanceolata, 18. Capartogramma crucicula, 19. Craticula cuspidata, 20. Craticula crucicula. 

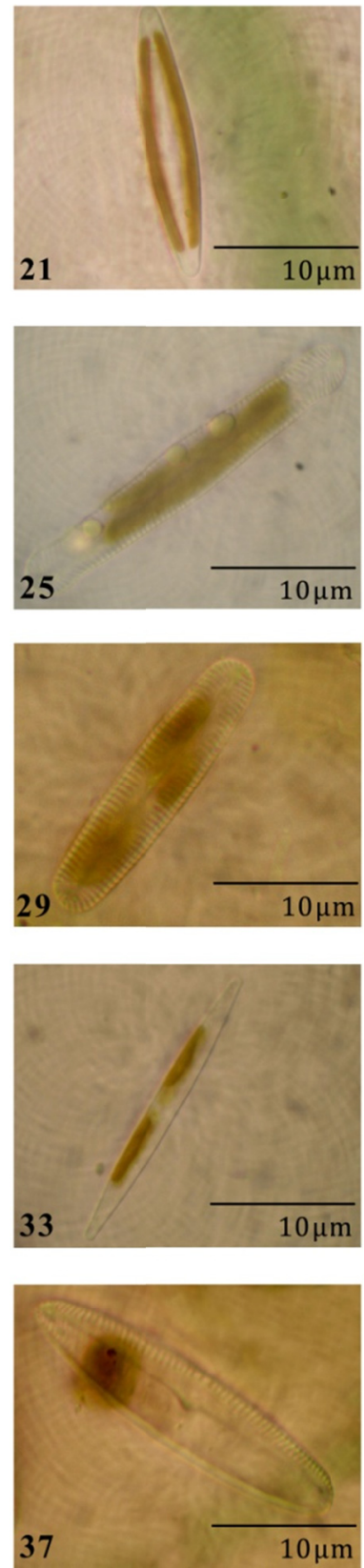
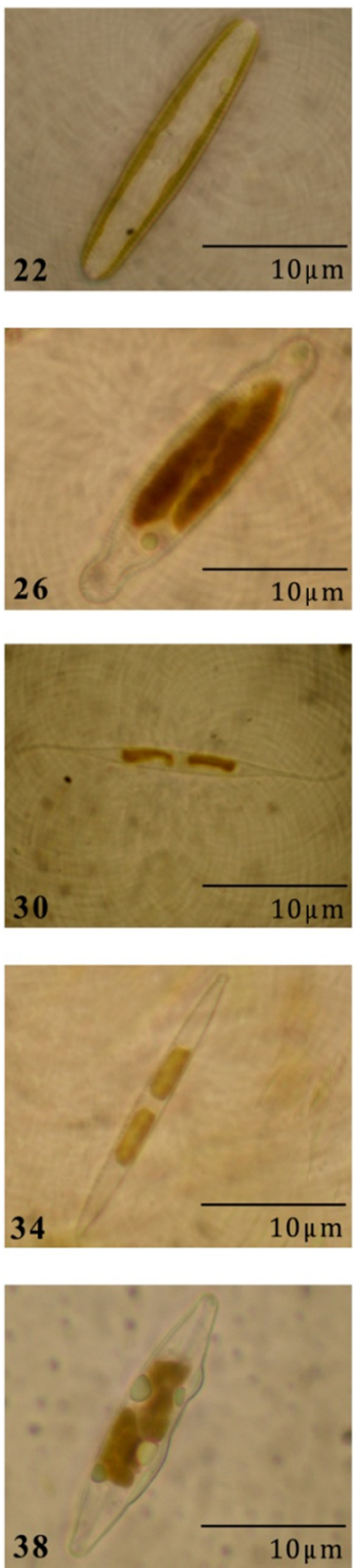
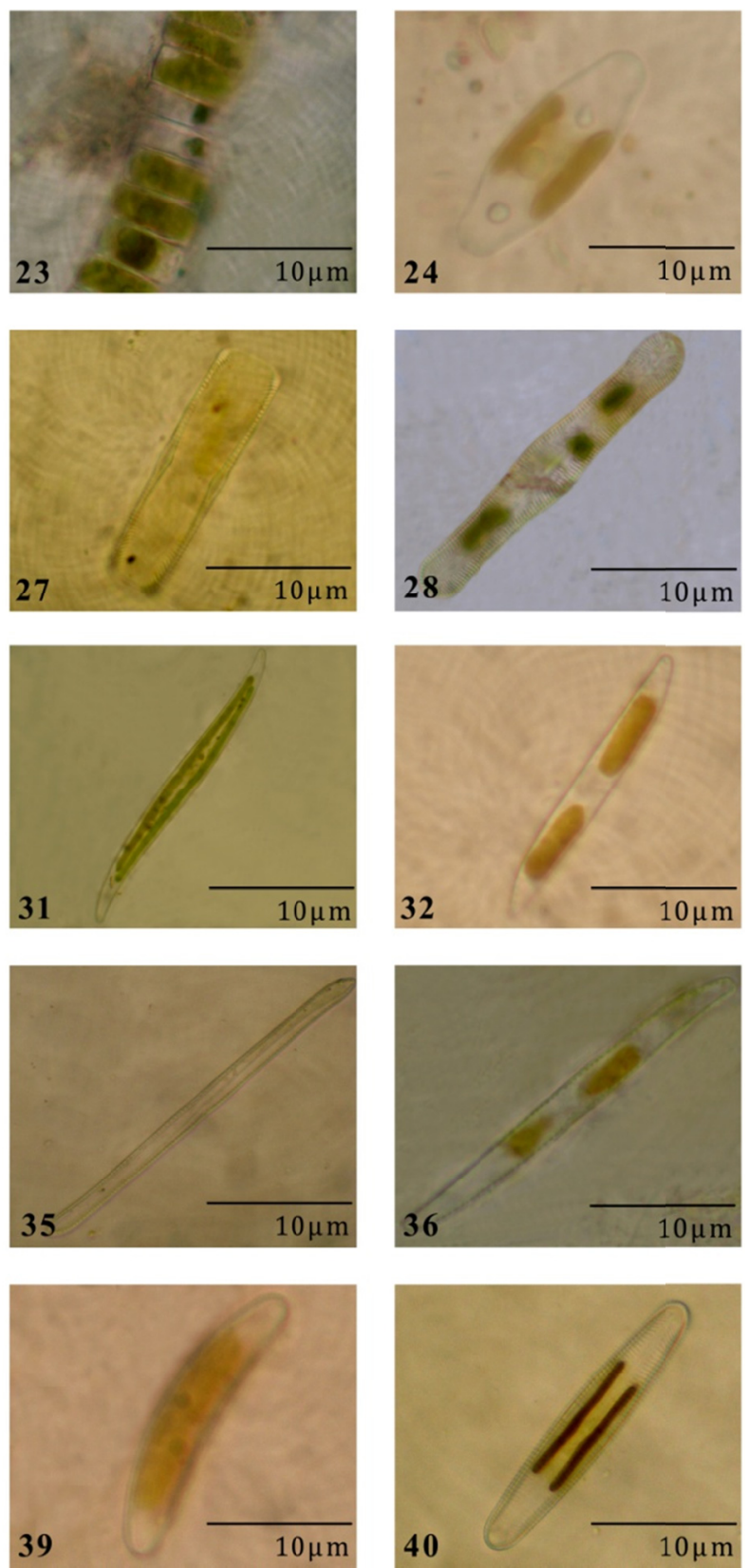

Figure 3. Diatoms $21-40$

21. Frustulia vulgaris, 22. Nedium affine, 23. Diadesmis confervacea, 24. Sellaphora ulna, 25. Pinnularia acrosphaeria, 26. Pinnularia biceps, 27. Pinnularia brauni, 28. Pinnularia gibba, 29. Pinnularia viridiformis, 30. Nitzchia reversa, 31. Nitzchia sigmoidea, 32. Nitzschia clausii, 33. Nitzschia filiformis, 34. Nitzschia liebertruthii, 35. Nitzschia nana, 36. Nitzschia obtusa, 37. Cymbella kolbei, 38. Gomphonema acuminatum, 39. Eunotia bilunaris, 40. Achanthidium minutissimum

Relative abundance of diatoms (Table 2; Figure 4) in percentage was; $5 \%$ from the order Melosirales, $10 \%$ from Fragilariales, $2 \%$ from Thalassiosirales, $2 \%$ from Thalassiophysales, $40 \%$ from Naviculales, $30 \%$ from Bacillariales, 5\% to from Cymbellales, 3\% from Eunotiales and 1\% from Achanthales. Navicula and Nitzschia were the dominant genera with 9 and 7 species respectively, followed by the genus Pinnularia with 5 species. 
Table 2. Relative abundance (\%) of occurrence of diatoms in Kuttanadu paddy fields in different regions, seasons and crop stages (R1-Upper Kuttanadu, R2-Lower Kuttanadu and R3-Kayal Lands; S1-Pre-monsoon (Puncha) crop and S2-Monsoon (Virippu) crop; G1-Seedling-growth-stage and G2-Mature-growth-stage)

\begin{tabular}{|c|c|c|c|c|c|c|c|c|c|c|c|c|c|c|}
\hline \multirow{3}{*}{$\begin{array}{l}\mathrm{N} \\
\mathrm{o}\end{array}$} & \multirow{3}{*}{ Diatom species } & \multicolumn{4}{|c|}{ R1 } & \multicolumn{4}{|c|}{$\mathrm{R} 2$} & \multicolumn{4}{|c|}{ R3 } & \multirow{3}{*}{$\begin{array}{l}\text { Abundance } \\
(\%)\end{array}$} \\
\hline & & \multicolumn{2}{|c|}{ S1 } & \multicolumn{2}{|l|}{ S2 } & \multicolumn{2}{|c|}{$\mathrm{S} 1$} & \multicolumn{2}{|c|}{ S2 } & \multicolumn{2}{|c|}{ S1 } & \multicolumn{2}{|c|}{ S2 } & \\
\hline & & $\begin{array}{l}\mathrm{G} \\
1\end{array}$ & $\begin{array}{l}\mathrm{G} \\
2\end{array}$ & $\begin{array}{l}\mathrm{G} \\
1\end{array}$ & $\begin{array}{l}\mathrm{G} \\
2\end{array}$ & $\begin{array}{l}\mathrm{G} \\
1\end{array}$ & $\begin{array}{l}\mathrm{G} \\
2\end{array}$ & $\begin{array}{l}\mathrm{G} \\
1\end{array}$ & $\begin{array}{l}\mathrm{G} \\
2\end{array}$ & $\begin{array}{l}\mathrm{G} \\
1\end{array}$ & $\begin{array}{l}\mathrm{G} \\
2\end{array}$ & $\begin{array}{l}\mathrm{G} \\
1\end{array}$ & $\begin{array}{l}\mathrm{G} \\
2\end{array}$ & \\
\hline & Order: Melosirales & & & & & & & & & & & & & \\
\hline 1 & Melosira varians Agardh & 1 & 1 & 4 & 6 & 1 & 1 & 6 & 4 & 1 & 1 & 3 & 3 & 26.67 \\
\hline \multirow[t]{2}{*}{2} & Melosira italica (Ehrenberg) Kutzing & 1 & 1 & 5 & 4 & 1 & 3 & 2 & 1 & 6 & 5 & 1 & 1 & 25.83 \\
\hline & Order: Thalassiosirales & & & & & & & & & & & & & \\
\hline \multirow[t]{2}{*}{3} & Cyclotella meneghinianaKutzing & 2 & 2 & 2 & 5 & 1 & 2 & 4 & 5 & 2 & 4 & 6 & 6 & 34.17 \\
\hline & Order: Fragilariales & & & & & & & & & & & & & \\
\hline 4 & Fragilaria capucina Desmazieres & 2 & 2 & 4 & 4 & 1 & 1 & 4 & 4 & 1 & 1 & 5 & 5 & 28.33 \\
\hline 5 & $\begin{array}{l}\text { Staurosira elliptica (Schumann) Williams \& } \\
\text { Round }\end{array}$ & 1 & 1 & 5 & 5 & 1 & 1 & 2 & 2 & 3 & 5 & 1 & 4 & 25.83 \\
\hline 6 & Synedra ulna(Nitzsch) Ehrenberg & & 3 & 3 & 3 & 1 & 2 & 4 & 4 & & & 2 & 2 & 20.00 \\
\hline \multirow[t]{2}{*}{7} & Synedra tabulata (C.Agardh) Kutzing & & 1 & 2 & 4 & 1 & 1 & 5 & 5 & 3 & 1 & 4 & 5 & 26.67 \\
\hline & Order: Thalassiophysales & & & & & & & & & & & & & \\
\hline \multirow[t]{2}{*}{8} & $\begin{array}{l}\text { Amphora pediculus (Kutzing) Grunowex. A. } \\
\text { Schmidt }\end{array}$ & & 1 & 3 & 4 & & 1 & 4 & 4 & 1 & 2 & 4 & 3 & 22.50 \\
\hline & Order: Naviculales & & & & & & & & & & & & & \\
\hline 9 & Navicula capitatoradiata Germain & 1 & 1 & 1 & 1 & 1 & 1 & 6 & 7 & 1 & 1 & 1 & 1 & 19.17 \\
\hline 10 & Navicula cuspidata (Kutzing) Kutzing & 1 & 1 & 1 & 1 & 1 & 1 & 1 & 1 & 1 & 1 & 1 & 1 & 10.00 \\
\hline 11 & Navicula ranomafenensis Kutzing & 1 & 1 & 1 & 4 & 1 & 1 & 2 & 2 & 1 & 1 & 2 & 4 & 17.50 \\
\hline 12 & Navicula reinhardtii (Grnow) Grunow & 1 & 1 & 7 & 6 & 1 & 1 & 3 & 3 & 1 & 1 & 5 & 2 & 26.67 \\
\hline 13 & Navicula salinarum Grunow & 1 & 1 & 3 & 4 & & & 3 & 5 & 1 & 1 & 1 & 1 & 17.50 \\
\hline 14 & Navicula pupula Kutzing & 1 & 1 & 1 & 1 & & 1 & 4 & 3 & & & 6 & 4 & 18.33 \\
\hline 15 & Navicula viridula (Kutzing) Ehrenberg & 1 & 1 & 4 & 5 & & & 6 & & & & 5 & 3 & 20.83 \\
\hline 16 & Navicula viridis (Nitzsch) Kutzing & 1 & 1 & 1 & 1 & & & 5 & & & & 4 & 4 & 14.17 \\
\hline 17 & Navicula lanceolata Ehrenberg & 2 & 1 & 1 & 1 & & & 4 & 5 & & & 7 & 8 & 24.17 \\
\hline 18 & Capartogramma crucicula Grunow (R.Ross) & 1 & & 1 & 1 & 2 & 2 & 2 & 2 & & 3 & 3 & 2 & 15.83 \\
\hline 19 & Craticula cuspidata (Kutzing) D.G.Mann & 1 & 1 & 1 & 1 & & & 3 & 5 & & & 3 & 3 & 15.00 \\
\hline 20 & Craticula halophila (Kutzing) D.G.Mann & 1 & 1 & 3 & 1 & 1 & 1 & 4 & 4 & 1 & & & & 14.17 \\
\hline 21 & Frustulia vulgaris (Thwaites) De Toni & 2 & 1 & 5 & 5 & 2 & 1 & 6 & 6 & 3 & 3 & 6 & 6 & 38.33 \\
\hline 22 & Nedium affine(Ehrn) Pfitzer & 1 & & 4 & 4 & 1 & 1 & 4 & 3 & 1 & 1 & 5 & 5 & 25.00 \\
\hline 23 & Diadesmus confervaceae (Kutzing) DG Mann & 2 & 1 & 5 & 5 & 1 & 1 & 6 & 6 & 1 & 1 & 8 & 5 & 35.00 \\
\hline \multirow[t]{2}{*}{24} & Sellaphora ulna(Nitzsch) Ehrenberg & 1 & & 3 & 5 & 2 & 2 & 3 & 4 & 2 & 2 & 4 & 1 & 24.17 \\
\hline & Order: Bacillariales & & & & & & & & & & & & & \\
\hline 25 & Pinnularia acrosphaeriaW Smith & 2 & 3 & 4 & 4 & 1 & 2 & 2 & 2 & 1 & 1 & 3 & 3 & 23.33 \\
\hline 26 & Pinnularia biceps W. Gregory & 3 & 2 & 2 & 4 & 1 & 1 & 4 & 4 & & & 2 & 2 & 20.83 \\
\hline 27 & Pinnularia brauni (Grunow) Cleve & 2 & 2 & 5 & 5 & 1 & 1 & 5 & 6 & & & 6 & 6 & 32.50 \\
\hline 28 & Pinnularia gibba Ehrenberg & 2 & 1 & 4 & 4 & 1 & 1 & 4 & 2 & 1 & 2 & 2 & 1 & 20.83 \\
\hline 29 & Pinnularia viridiformis $\mathrm{Krammer}$ & 2 & 1 & 3 & 3 & 1 & 1 & 2 & 2 & 1 & 1 & 2 & 2 & 17.50 \\
\hline 30 & Nitzschia reversa W Smith & 3 & 3 & 4 & 6 & 1 & 1 & 6 & 7 & 1 & 2 & 7 & 8 & 40.83 \\
\hline 31 & Nitzschia sigmoidea (Nitzsch) W. Smith & 1 & 1 & 1 & 4 & 1 & 1 & 4 & 5 & 1 & 1 & 6 & 7 & 27.50 \\
\hline 32 & Nitzschia clausiiHantzsch & 1 & 2 & 8 & 6 & 1 & 1 & 6 & 7 & 1 & 1 & 7 & 8 & 40.83 \\
\hline 33 & Nitzschia filiformis (W Smith) Vanurk & 1 & 1 & 3 & 5 & 1 & 2 & 2 & 1 & 1 & 1 & 1 & 1 & 16.67 \\
\hline 34 & Nitzschia linearis W Smith & 1 & 1 & 1 & 1 & 1 & 1 & 1 & 1 & 1 & 1 & 1 & 3 & 11.67 \\
\hline 35 & Nitzschia nana Grunow & 2 & 2 & 5 & 4 & 1 & 1 & 7 & 7 & 2 & 1 & 4 & 6 & 35.00 \\
\hline 36 & Nitzschia obtusa Rabenhorst & 2 & 2 & 1 & 1 & 1 & 1 & 2 & 2 & 2 & 2 & 5 & 5 & 21.67 \\
\hline
\end{tabular}




\begin{tabular}{|c|c|c|c|c|c|c|c|c|c|c|c|c|c|c|}
\hline \multirow{3}{*}{$\begin{array}{l}\mathrm{N} \\
\mathrm{o}\end{array}$} & \multirow{3}{*}{ Diatom species } & \multicolumn{4}{|l|}{ R1 } & \multicolumn{4}{|l|}{$\mathrm{R} 2$} & \multicolumn{4}{|l|}{$\mathrm{R} 3$} & \multirow{3}{*}{$\begin{array}{l}\text { Abundance } \\
(\%)\end{array}$} \\
\hline & & \multicolumn{2}{|l|}{ S1 } & \multicolumn{2}{|l|}{$\mathrm{S} 2$} & \multicolumn{2}{|l|}{ S1 } & \multicolumn{2}{|l|}{ S2 } & \multicolumn{2}{|l|}{ S1 } & \multicolumn{2}{|l|}{ S2 } & \\
\hline & & $\begin{array}{l}\mathrm{G} \\
1\end{array}$ & $\begin{array}{l}\mathrm{G} \\
2\end{array}$ & $\begin{array}{l}\mathrm{G} \\
1\end{array}$ & $\begin{array}{l}\mathrm{G} \\
2\end{array}$ & $\begin{array}{l}\mathrm{G} \\
1\end{array}$ & $\begin{array}{l}\mathrm{G} \\
2\end{array}$ & $\begin{array}{l}\mathrm{G} \\
1\end{array}$ & $\begin{array}{l}\mathrm{G} \\
2\end{array}$ & $\begin{array}{l}\mathrm{G} \\
1\end{array}$ & $\begin{array}{l}\mathrm{G} \\
2\end{array}$ & $\begin{array}{l}\mathrm{G} \\
1\end{array}$ & $\begin{array}{l}\mathrm{G} \\
2\end{array}$ & \\
\hline & Order: Cymbellales & & & & & & & & & & & & & \\
\hline 37 & Cymbella kolbei Hustedt & 1 & 1 & 1 & 1 & 1 & 1 & 1 & 1 & 1 & 1 & 1 & 1 & 10.00 \\
\hline \multirow[t]{2}{*}{38} & Gomphonema acuminatumEhr & & 1 & 1 & 1 & 1 & 1 & 1 & 1 & & 1 & 2 & 2 & 10.00 \\
\hline & Order: Eunotiales & & & & & & & & & & & & & \\
\hline \multirow[t]{3}{*}{39} & Eunotia bilunaris (Ehrenberg) Mills & 2 & 2 & 3 & 4 & 2 & 1 & 1 & 1 & 1 & 2 & 2 & 2 & 19.17 \\
\hline & Order: Achnanthales & & & & & & & & & & & & & \\
\hline & $\begin{array}{l}\text { Achnanthidium minutissimum(Kutzing) } \\
\text { Czarnecki }\end{array}$ & & & & & 1 & 1 & 1 & 1 & & & & & 3.33 \\
\hline 40 & Total No. of species & 35 & 36 & 39 & 39 & 33 & 35 & 40 & 38 & 29 & 30 & 38 & 38 & \\
\hline
\end{tabular}

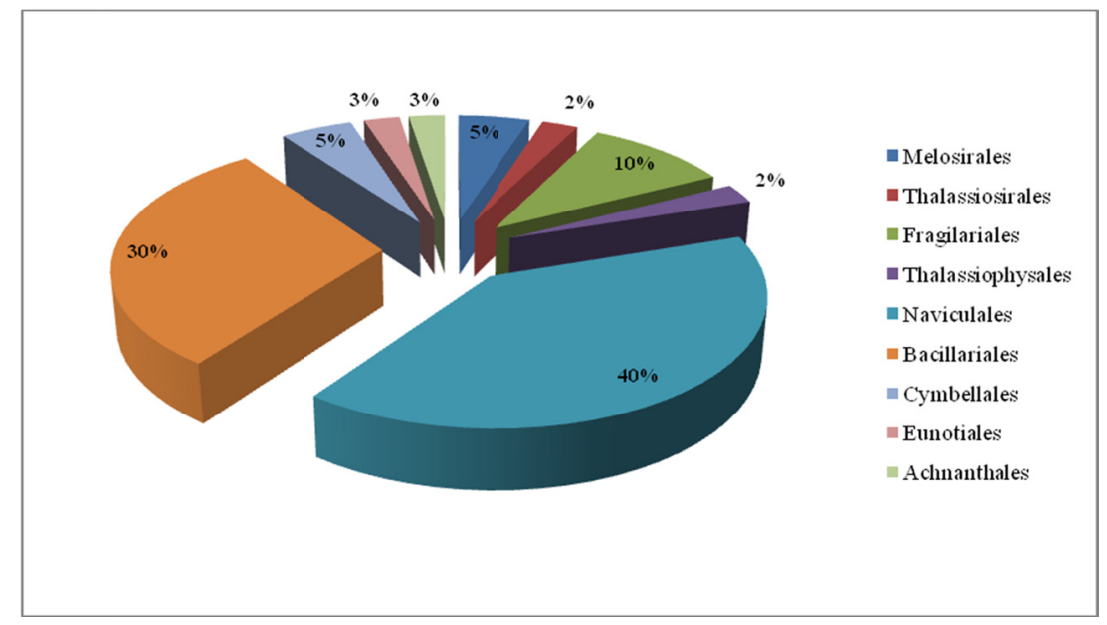

Figure 4. Relative abundance of various orders of Diatoms in Kuttanadu

\subsection{Seasonal Variations in the Number of Species and Total Isolates}

The highest number of species of diatoms (40 species) and the highest number of total isolates (355) were observed at panicle-growth-stage in the Puncha cultivation season of Kayal lands, and the lowest number of species (25 species) and the lowest total isolates (69) was also found at the panicle-growth-stage of in the 'Virippu' cultivation of Kayal lands.

The most abundant species in Upper Kuttanadu and Lower Kuttanadu was found to be Pinnularia brauni, but that of 'Kayal' lands was Nedium affine. In the 'Puncha' season Pinnularia braunii was found to be the most abundant species in Kuttanadu but that in the 'Virippu' season was Frustulia vulgaris.

\subsection{Ecological Characteristics of the diatom community in Kuttanadu}

The ecological characteristics included in the present study were relative abundance, species richness, species evenness and diversity index. The relative abundance of all the 40 algal species calculated from 120 soil samples belonging to the three different regions of Kuttanadu was found out; Nitzchia clausii $(74.17 \%)$, showed highest relative abundance followed by Frustulia vulgaris (72.5\%) and Nedium affine (70\%). The least abundant algae in the whole Kuttanadu were Staurosira construens (20.83\%). The most abundant species in 'Upper Kuttanadu' and 'Lower Kuttanadu' was found to be Pinnularia brauni with relative abundances $85 \%$ and $82.5 \%$ respectively and that in 'Kayal' lands was Nedium affine with relative abundance $82.5 \%$.

Species richness and Diversity Index of diatoms (Table 3) varied from 0.884-1.158 in different regions of Kuttanadu during the two crop seasons and crop stages. The species richness was found maximum (1.158) in 'Lower Kuttanadu', during 'Virippu' season at panicle-growth-stage. Minimum species richness (0.884) was noticed in 'Kayal' lands during 'Puncha' cultivation at seedling stage. The species diversity index of diatoms in Kuttandu was found varying from $1.06-1.17$ in different regions of Kuttanadu during the two crop seasons and crop stages. The diversity index was found maximum (1.17) in 'Lower Kuttanadu', during 'Virippu' season at 
seedling stage. Minimum diversity index (1.06) was noticed in 'Kayal' lands during 'Puncha' cultivation season at seedling-growth-stage of the crop. The species evenness showed very slight variation from $0.315-0.317$ in different regions of Kuttanadu during the two crop seasons and crop-growth-stages.

Table 3. Species richness and diversity index of diatoms in different seasons and vegetations (UK - upper Kuttanadu; LK - lower Kuttanadu; KL - Kayal lands; R1-Upper Kuttanadu, R2-Lower Kuttanadu and R3-Kayal Lands; S1-Pre-monsoon (Puncha) crop and S2-Monsoon (Virippu) crop; G1-Seedling-growth-stage and G2-Mature-growth-stage)

\begin{tabular}{|c|c|c|c|c|c|c|}
\hline Region & Season & Growth Stage & No. of Species & Total Isolates & Sps rich & Div.Ind \\
\hline \multirow{4}{*}{ UK } & \multirow{2}{*}{$\mathrm{S} 1$} & G1 & 35 & 51 & 1.066 & 1.12 \\
\hline & & $\mathrm{G} 2$ & 36 & 50 & 1.097 & 1.13 \\
\hline & \multirow{2}{*}{$\mathrm{S} 2$} & G1 & 39 & 116 & 1.188 & 1.16 \\
\hline & & G2 & 39 & 134 & 1.188 & 1.16 \\
\hline \multirow{4}{*}{ LK } & \multirow{2}{*}{$\mathrm{S} 1$} & G1 & 33 & 37 & 1.006 & 1.10 \\
\hline & & $\mathrm{G} 2$ & 35 & 43 & 1.066 & 1.12 \\
\hline & \multirow{2}{*}{$\mathrm{S} 2$} & G1 & 40 & 142 & 1.219 & 1.17 \\
\hline & & $\mathrm{G} 2$ & 38 & 135 & 1.158 & 1.15 \\
\hline \multirow{4}{*}{ KL } & \multirow{2}{*}{$\mathrm{S} 1$} & G1 & 29 & 44 & 0.884 & 1.06 \\
\hline & & $\mathrm{G} 2$ & 30 & 51 & 0.914 & 1.07 \\
\hline & \multirow{2}{*}{$\mathrm{S} 2$} & G1 & 38 & 138 & 1.158 & 1.15 \\
\hline & & $\mathrm{G} 2$ & 38 & 136 & 1.158 & 1.15 \\
\hline
\end{tabular}

Sps. Rich - species richness; Div.Ind - Diversity Index.

\subsection{Physico-Chemical Characteristics of the Soils as per Ray et al. (2014)}

Variations in $\mathrm{pH}$, total organic carbon $(\mathrm{C})$, total/ Kjeldal nitrogen $(\mathrm{N})$, plant available phosphorus $(\mathrm{P})$, potassium $(\mathrm{K})$, calcium $(\mathrm{Ca})$ and magnesium $(\mathrm{Mg})$ studied in relation to crop seasons and different stages of paddy growth in the three different regions of Kuttanadu are given in Table 4.

Table 4. Average soil chemical characteristics of wetland paddy fields of Kuttanadu (Ray et al., 2014)

\begin{tabular}{|c|c|c|c|c|c|c|c|c|c|c|}
\hline \multirow[b]{2}{*}{ No } & \multirow[b]{2}{*}{ Location } & \multirow{2}{*}{$\begin{array}{l}\text { Crop } \\
\text { season }\end{array}$} & \multirow{2}{*}{$\begin{array}{l}\text { Growth } \\
\text { stage }\end{array}$} & \multirow{2}{*}{$\begin{array}{l}\text { Average } \\
\mathrm{p}^{\mathrm{H}}\end{array}$} & \multirow{2}{*}{$\begin{array}{l}\text { Average } \\
\text { TN (\%) }\end{array}$} & \multirow{2}{*}{$\begin{array}{l}\text { Average } \\
\text { OC (\%) }\end{array}$} & \multicolumn{4}{|c|}{ Average available nutrients $(\mathrm{kg} / \mathrm{ha})$} \\
\hline & & & & & & & $\mathrm{P}$ & $\mathrm{K}$ & $\mathrm{Ca}$ & $\mathrm{Mg}$ \\
\hline 1 & UK & Puncha & Seedling & 3.94 & 0.429 & 3.07 & 10.86 & 998.6 & 3858.8 & 788.8 \\
\hline 2 & UK & Puncha & Panicle & 3.88 & 0.392 & 4.06 & 18.26 & 656.9 & 2378.6 & 512.2 \\
\hline 3 & UK & Virippu & Seedling & 3.40 & 0.289 & 0.94 & 129.00 & 298.7 & 441.4 & 177.7 \\
\hline 4 & UK & Virippu & Panicle & 3.65 & 0.459 & 2.63 & 110.00 & 496.5 & 758.2 & 205.5 \\
\hline 5 & LK & Puncha & Seedling & 4.00 & 0.413 & 3.97 & 7.14 & 855.0 & 1842.4 & 785.8 \\
\hline 6 & LK & Puncha & Panicle & 4.29 & 0.374 & 3.54 & 25.34 & 389.2 & 2841.7 & 840.2 \\
\hline 7 & LK & Virippu & Seedling & 3.74 & 0.370 & 1.68 & 109.00 & 316.7 & 1078.0 & 321.9 \\
\hline 8 & LK & Virippu & Panicle & 3.70 & 0.520 & 2.16 & 95.00 & 408.9 & 1437.4 & 377.4 \\
\hline 9 & $\mathrm{KL}$ & Puncha & Seedling & 4.03 & 0.390 & 3.28 & 9.60 & 391.2 & 1249.6 & 748.5 \\
\hline 10 & $\mathrm{KL}$ & Puncha & Panicle & 4.61 & 0.435 & 3.70 & 9.46 & 396.0 & 2331.8 & 992.1 \\
\hline 11 & KL & Virippu & Seedling & 4.05 & 0.400 & 2.49 & 129.00 & 440.9 & 1466.6 & 407.3 \\
\hline 12 & KL & Virippu & Panicle & 4.23 & 0.431 & 2.68 & 58.00 & 408.3 & 1618.7 & 399.9 \\
\hline
\end{tabular}

TN - Total nitrogen; OC - Total organic carbon.

\subsection{SEM Analyses of Soil Samples for Diatoms}

SEM analyses of the Kuttanadu soil samples revealed the presence of seven species of diatoms (Figure 5). Randomly selected six soil samples from the three regions of Kuttanadu were analyzed for diatoms. Cyclotella meneghiniana Kutzing, and Achnanthidium atomus (Hustedt) Monnier, Lange- Bertalot and Ector were reported from 'Upper Kuttanadu' samples. Aulocoseira granulata (Ehrenberg) Simonsen, and Diadesmisconfervaceae 
Kutzing and Eunotiasp were reported from 'Lower Kuttanadu' soil samples. Fragilariformavirescens Ralfs (Williams) and Rounds and Pinnularia sp. were observed from 'Kayal' land samples.
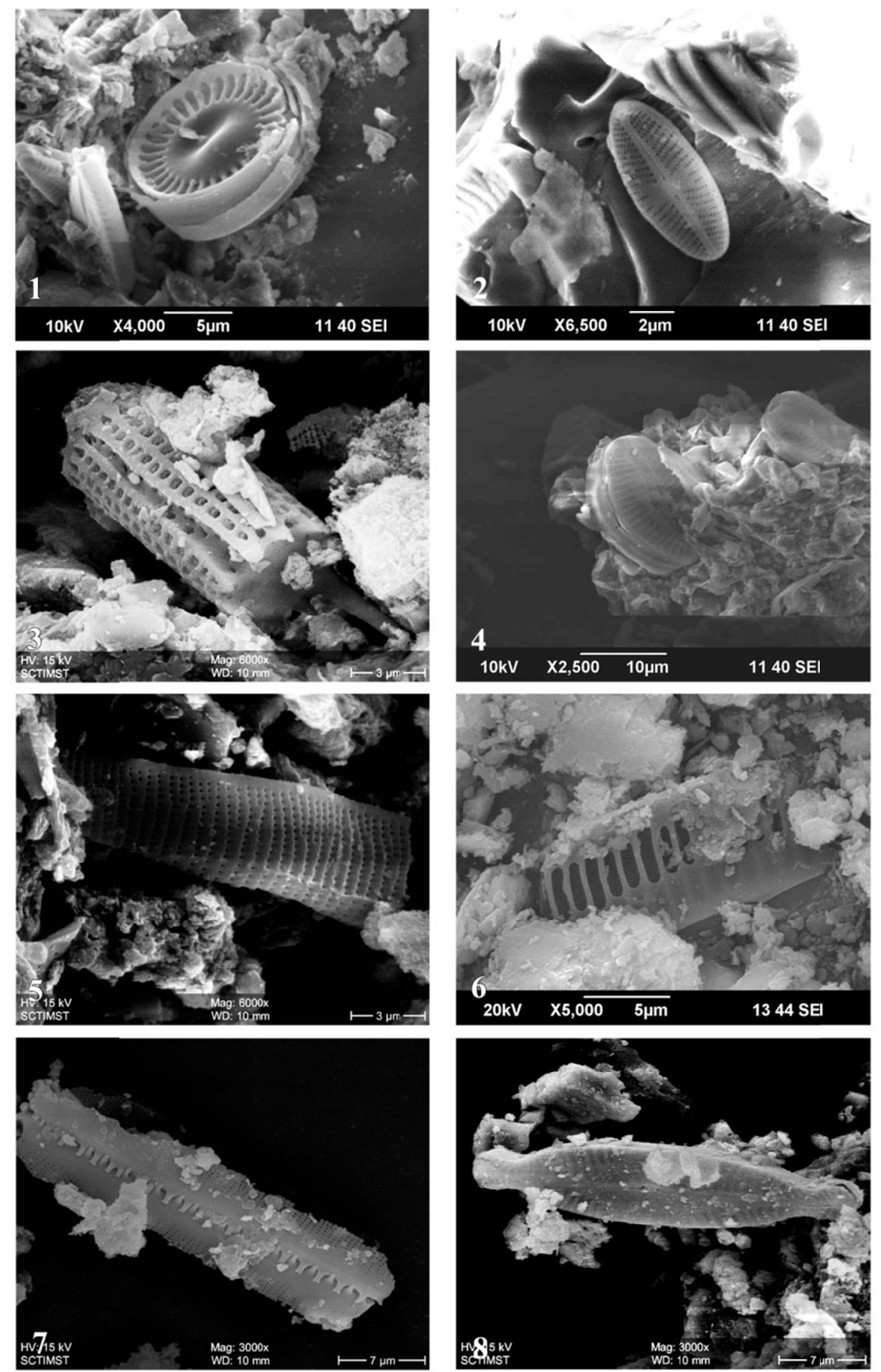

Figure 5. SEM pictures of Diatoms 1 - 8

1. Cyclotella meneghiniana Kutzing, 2. Achnanthidium atomus (Hustedt) Monnier, Lange -Bertalot and Ector, 3. Aulocoseira granulata (Ehrenberg) Simonsen, 4. Diadesmis confervaceae Kutzing, 5. Eunotia macroglossa R. C. Furey, R. L. Lowe and J. R. Johansen, 6. Pinnularia - showing Chambered striae, 7. Fragilariforma virescens Ralfs (Williams) and Rounds 8. Pinnularia $s p$. 


\section{Discussion}

Soil is a complex heterogeneous environment (El-Gamal, 2008). Human influences affect its physical, chemical, and biological characteristics. In general, high diatom diversity is used as an indicator to anthropogenic disturbance and environmental contaminations (Vacht, Puusepp, Koff, \& Reitalu, 2014). Among algae, diatom communities are considered as indicators of both organic and anthropogenic pollutions. In the present study, diatom diversity was reported to be high in almost all regions, seasons and growth stages, which indicated that the disturbed nature of this agro-ecosystem. In the current investigation, the highest number of diatom species, total isolates, species richness and diversity index were observed in the Lower Kuttanadu paddy soils in the seedling stage (early monsoon or pre-monsoon) of Virippu cultivation, which gradually decreased towards panicle-growth-stage (the monsoon season).

Comparatively lower diatom diversity is observed in non-monsoon crop season (Puncha cultivation) than in the monsoon crop (Virippu), which showed the influence of climate in determining the diatom density of Kuttanadu wetland paddy soils. Ecological conditions have prominent role in determining the algal diversity (Lin, Chou \& Wu, 2013). Redekar and Wagh (2000), Pareek et al. (2011), Muralidhar and Murthy (2014), and Jayabhaye (2010) noticed that diatom diversity reaches maximum during monsoon or pre-monsoon months and minimum during summer. Contradictory to these findings, Muruganantham et al. (2012), observed maximum diversity of diatoms in summer.

According to Metting (1981), Neustupa and Skaloud (2008) and Lames -Da-Silva, Branco \& Necchi- Junior, (2010) light play a significant role in the growth and differentiation of algae. In the present study, highest diatom diversity and abundance was observed in seedling stage of second cultivation (Virippu), when light is abundant due to less canopy of the crop plant. Roger and Reynaud (1982) also observed that diatom community increases in paddy fields during seedling stage of the crop. Lin et al. (2013), Fujita and Ohtsuka (2005), also supported this view that diatom cell density reaches the highest number just after transplanting of rice and thereafter decreases.

In the current investigation, the Diatom species richness and diversity index were found to be correlated (Pearson's correlation coefficient) to soil parameters such as $\mathrm{pH}, \mathrm{N}, \mathrm{P}, \mathrm{K}, \mathrm{Ca}$ and $\mathrm{Mg}$ of different regions of Kuttanadu at different crop-growth-stages and crop-seasons. A variety of physical and biological factors influence the distribution and diversity of diatoms. Comerton and Houghton (1978) observed that inorganic nutrients stimulates or suppress algal growth in the soil. Nutrient concentration is the most important factor that influences the distribution and abundance of Diatom species (Broady, 1979; Mohan, Shukla, Patil, Shetye, \& Kerkar, 2011). In the present study, highest Diatom diversity was observed in 'Lower Kuttanadu' soil. Soil factors, vegetation and topography affect the algal biomass (Patrick, Groffman, Driscoll, Fahy, \& Siccama, 2001), especially diatoms (Venkitachalapathy \& Karthikeyan, 2013). Soil of 'Lower Kuttanadu' region is clay loams characterized by a fairly high amount of nitrogen. Diatom diversity, community behaviour and composition are influenced by nitrates (Muralidhar \& Murthy, 2014). But in the present study, nitrates in Kuttanadu soils are not found to be correlated to any diversity parameters of Diatoms there. On the other hand, species richness and diversity index showed a significant positive correlation to phosphorus. This is in agreement with Comerton and Houghton (1978) that phosphorus increases the algal count, especially that of Diatoms in natural environment.

In the present study, species richness and diversity index showed significant negative correlation with $\mathrm{pH}$. This is in agreement with the observations of Muralidhar and Murthy (2014) that Diatoms are sensitive to $\mathrm{pH}$ and salinity. Lukesova (2001) observed that Diatoms prefer neutral to alkaline pH. But, Diatoms showed high diversity in Kuttanadu soils, where $\mathrm{pH}$ ranges from 3.4 to 4.6, which is in agreement with the observations of Muralidhar and Murthy (2014) that pH has no role in the periodicity and density of Diatoms in soils.

\section{Conclusion}

Overall, the present analysis of the ecological characteristics of Diatoms of Kuttanadu wetland paddy fields in relation to soil regions, crop-seasons and paddy-growth-stages appears quite useful to the exploration of the economic potentials of many individual species of Diatoms. Since many of the diatoms observed in this investigation are found quite specific to soils or seasons or crop growth stages, further investigations on the environment factors contributing to limited occurrence of them in specific locations alone, would enable identification of them as bio-indicators of diverse environmental conditions.

\section{Acknowledgements}

Authors wish to acknowledge facilities provided by Laboratory of the Sophisticated Test and Instrumentation Centre (STIC), University of Science and Technology, Cochin for SEM analyses of diatoms in soils.

\section{References}


Bray, R. H., \& Kurtz, L. T. (1945). Determination of total, organic, and available forms of phosphorus in soils. Soil Science, 59, 39-45. http://dx.doi.org/10.1097/00010694-194501000-00006

Broady, P. A. (1979). Quantitative studies on the terrestrial algae of Signy Island, South Orkney Islands. British Antartic Survey Bulletin, 47, 31-41.

Comerton, M., \& Houghton, J. A. (1978). The effects of fertilizers on the algal flora of peat. Proceedings of the Royal Irish Academy, 8, 233-245.

Demirbas, A., \& Demirbas, M. F. (2011). Importance of algae oil as a source of biodiesel. Energy Conservation and Management, 52, 163-170. http://dx.doi.org/10.1016/j.enconman.2010.06.055

Dey, H. S, Tayung, K., \& Bastia, A. K. (2010). Occurence of nitrogen fixing Cyanobacteria in local rice fields of Orissa, India. Ecoprint, 17, 77-85.

El-Gamal, A. D., Nady, A., Ghanem, E., Eisha, Y. E. 1- Ayouty., \& Shehatha, E. F. (2008). Studies on soil algal flora in Kafrel- sheikh Governnorate Egypt. Egyptian Journal of Phycology, 9, 1-23.

Fujitha, Y., \& Ohtsuka, T. (2005). Diatoms from paddy fields in northern Laos. Diatom, 21, 71-89.

Gandhi, H. P. (1956). A Preliminary account of the soil diatom flora of Kolhapur. The Journal of Indian Botanical Society, 35, 402-408.

Guiry, M. D., \& Guiry, G. M. (2012). Worldwide electronic publication, National University of Ireland, Galway. Retrieved from http://www.algaebase.org

Jackson, M. L. (1973). Soil Chemical analysis (pp. 487-498). Prentice Hall of India, Private limited, NewDelhi, India.

Jayabhaye,U. M. (2010). Studies on Phytoplankton diversity in Sawana Dam, Maharashtra India, Shodh Samiksha aur Mulyankan. International Research Journal, 11, 11-12.

Jena, M., Ratha, S. K., \& Adhikary, S. P. (2006). Diatoms (Bacillariophyceae) from Orissa state and Neighbouring regions, India. Algae, 21(4), 377-392. http://dx.doi.org/10.4490/ALGAE.2006.21.4.377

Kesic, K., Tuney, I., Zerben, D., Guden, M., \& Sukatar, A. (2013). Morphological and molecular identification of pinnate diatoms isolated from Urla, Izmil, Coast of the Aegian Sea. Turkish Journal of Biology, 37, 530-537. http://dx.doi.org/10.3906/biy-1205-40

Lames-Da-Silva, N. M., Branco, L. H. Z., \& Necchi- Junior, O. (2010). Corticolous green algae from tropical forest remnants in the north west region of Sao Paulo State, Brazil. Revista Brasil Botany, 33, 215-226.

Lin, C., Chou, T., \& Wu, J. (2013). Biodiversity of soil algae in the farmlands of mid-Taiwan. Biological Studies, 54, 4. http://dx.doi.org/10.1186/1999-3110-54-41

Lukesova, A. (2001). Soil Algae in Brown coal and Lignite Post mining areas in Central Europe (Czech Republic and Germany). Restoration Ecology, 9, 341-350. http://dx.doi.org/10.1046/j.1526-100X.2001.94002.x

Mazumder, A., Govili., Ghosh, A. K., \& Ravindra, R. (2012).Significant research on diatom in Antartica Lake during last decade. Journal of Algal Biomass Utilization, 3(4), 74-79.

McGinnis, K. M., Dempster, T. A., \& Sommerfeld, M. R. (1997). Characterization of the growth and lipid content of the diatom Chaetoceros muelleri. Journal of Applied Phycology, 9(1), 19-24. http://dx.doi.org/10.1023/A:1007972214462

Metting, B. (1981). The Systematics and Ecology of Soil Algae. The Botanical Review, 147(2), 195-312. http://dx.doi.org/10.1007/BF02868854

Mohan, R. R., Shukla, S. K.., Patil, S. M., Shetye, S. S., \& Kerkar, K. K. (2011). Diatoms from surface sediments of Enderby basin of Indian Sector of Southern Ocean. Journal Geological Society of India, 78, 36-44. http://dx.doi.org/10.1007/s12594-011-0065-9

Mohan, S. K. (1987). Bacillariophyceae of two tropical South Indian lakes of Hyderabad. Botanical Bulletin, Academia Sinica, 28, 13-24.

Muruganantham, P., Gopalakrishnan, T., Chandrasekharan, R., \& Jayachandran, S. (2012). Seasonal variations and diversity of Planktonic diatoms of Muthupet and Aarukattuthura, South east coast of India. Advances in Applied Science and Research, 3(2), 919-929.

Nautiyal, P., \& Verma, J. (2009). Taxonomic Richness and diversity of the epilithic diatom flora of the two biogeographic regions of Indian sub-continent. Bulletin of the National Institute of Ecology, 19, 1-4. 
Neustupa, J., \& Skaloud, P. (2008). Diversity of sub-aerial algae and Cyanobacteria on tree bark in tropical mountain habitats. Biologia, 63, 806-812. http://dx.doi.org/10.2478/s11756-008-0102-3

Pandey, U. C., \& Pandey, D. C. (1980). Diatom flora of Allahabad (India)-1. Proc. Indian Natn. Sci. Acad. B46, 3, 350-355.

Pareek, R., Singh, G. P., \& Singh, R. (2011). Some fresh water diatoms of Galta Kund, Jaipur,India. Journal of Soil Science and Environmental Management, 2(4), 110-116.

Patrick, J. B., Groffman, P. M., Driscoll, C. T., Fahy, T. J., \& Siccama, T. G. (2001). Plant Soil Microbial interactions in a northern hard wood forest. Ecology, 82(4), 965-978.

Pillai, V. K., Ponniah, A. G., Vincent, D., \& David Raj, I. (1983). Acidity in Vembanadu Lake causes fish mortality, Marine Fisheries Information Series, T \& E Series No 53.

Ray, J. G., Dhanya, V., \& Binoy, T. T. (2014). Globally unique Kuttanadu wetland paddy soil of South India: Soil fertility in relation to seasons and different stages of crop. International journal of agriculture photon, 125 , 296-304.

Redekar, P. D., \& Wagh, A. B. (2000). Planktonic diatoms of the Zuari estuary, Goa (West Coast of India). Seaweed Research Utilization, 22(1 \& 2), 107-112.

Roger, P. A., \& Reynaud, P. A. (1982). Free living blue green algae in tropical soils. In Y. Dommergues \& H. Diem (eds) Microbiology of tropical soils and plant productivity. Martinus Nijhoff Publisher La Hague, 147- 168. http://dx.doi.org/10.1007/978-94-009-7529-3_5

Sarode, P. T., \& Kamat, N. D. (1984). Fresh water diatoms of Maharashtra (p. 338).

Serieyssol, K., Chatelard, S., \& Cubizolle, H. (2010). Diatom fossils in mires: a protocol for extraction, preparation and analysis in palaeoenvironmental studies, Mires and Peat, 7(12), 1-11.

Shannon, C. E., \& Wiener, W. (1949). The Mathematical Theory of Communication. University of Illinois, press Urbana.

Souffreau, C., Vanormelingen, P., Verleyen, E., Sabbe, K., \& Vyverman, W. (2010). Tolerance of benthic diatoms from temperate aquatic and terrestrial habitats to experimental desiccation and temperature stress. Phycologia, 49(4), 309-324. http://dx.doi.org/10.2216/09-30.1

Sudhikumar, A. V., Mathew, M. J., Sunish, E., \& Sebastian, P. A. (2005). Seasonal variation in spider abundance in Kuttanadu rice agroecosystem, Kerala, India (Araneae). European Arachnology, Acta zoologica bulgarica, l(1), 181-190.

Taylor, J. C., Archibald, C. G. M., \& Harding, W. R. (2007). An illustrated guide to some common diatom species from South Africa (WRC Report TT 282/07).

Vacht, P., Puusepp, L., Koff, T., \& Reitalu, T. (2014). Variability of riparian soil diatom communities and their potential as indicators of anthropogenic disturbances. Estonian Journal of Ecology, 63(3), 168-184. http://dx.doi.org/10.3176/eco.2014.3.04

Van de Vijver, \& Beyens, L. (1998). A preliminary study on the soil diatom assemblages from Ile de la Possession (Crozet, Subantartica). Polar Biology, 25, 721-729.

Venkatachalapathy, R., \& Karthikeyan , P. (2013). Benthic diatoms in river influenced by Urban pollution, Bhavani region, Cauvery river, South India. International Journal of Innovative technology and exploring engineering, 2(3), 206-210.

Venkataraman, K. (2005). Coastal and marine biodiversity of India. Indian Journal of Marine Sciences, 34(1), 57-75.

Vijayan, D., \& Ray, J. G. (2015b). Ecology and diversity of cyanobacteria in Kuttanadu. Paddy wetlands, Kerala, India. American Journal of Plant Sciences, 6, 2924-2938. http://dx.doi.org/10.4236/ajps.2015.618288

Vijayan, D., \& Ray, J. G. (2015a). Green algae of a unique tropical wetland, Kuttanadu, Kerala, India, in relation to soil regions, seasons and paddy growth stages. International Journal of Science, Environment and Technology, 4(3), 770-803.

Walkley, A., \& Black, I. A. (1934). An examination of Degtjareff Method for determining soil organic matter and a proposed modification of the chronic acid titration method. Soil Science, 37, 29-37. http://dx.doi.org/10.1097/00010694-193401000-00003 
Whittaker, R. H. (1977). Evolution of species diversity in land community. In M. K. Heeht, W. C. Stee \& B. Wallace (Eds.), Evolutionary Biology. Plenum, New York. http://dx.doi.org/10.1007/978-1-4615-6953-4_1

Wanga, X. W., Jun-Rong Lianga, Chun-Shan Luoa, Chang-Ping Chena, b, \& Ya-Hui Gao (2014). Biomass, total lipid production, and fatty acid composition of the marine diatom Chaetoceros muelleri in response to $\begin{array}{llllll}\text { different } \mathrm{CO} 2 \text { levels. } & \text { Bioresource }\end{array}$ http://dx.doi.org/10.1016/j.biortech.2014.03.012

Xue, J., Ying-Fang Niu, Tan Huang, Wei-Dong Yang, Jie-Sheng Liu, \& Hong-Ye, Li (2015) Genetic improvement of the microalga Phaeodactylum tricornutum for boosting neutral lipid accumulation. Metabolic Engineering, 27, 1-9. http://dx.doi.org/10.1016/j.ymben.2014.10.002

\section{Copyrights}

Copyright for this article is retained by the author(s), with first publication rights granted to the journal.

This is an open-access article distributed under the terms and conditions of the Creative Commons Attribution license (http://creativecommons.org/licenses/by/3.0/). 